\title{
MediSmart: Better Health with IOT Based Med Box
}

\author{
Vaibhavi G. Raut ${ }^{1}$, Praparna Moharana ${ }^{2}$, Tanya Patil ${ }^{3}$, Shantanu Ghanekar ${ }^{4}$, Prof. Swati A. Joshi ${ }^{5}$ \\ Student, Department of Computer Engineering, Sinhgad College of Engineering, Pune, India ${ }^{1,2,3,4}$ \\ Associate Professor, Department of Computer Engineering, Sinhgad College of Engineering, Pune, India ${ }^{5}$
}

\begin{abstract}
Multiple surveys indicate that about $65 \%$ of Adults are elderly people and are suffering from chronic diseases like diabetes, cholesterol, arthritis, osteoporosis, dementia, blindness, Alzheimer and so on. Also, the youngsters have fallen prey to the busy and hectic lifestyle. This busy lifestyle has made them forget to take their medicines on time, which is essential for their healthy recovery from their diseases. MediSmart proposes a solution to this distress. It is a smart, IOT based medicine or pillbox that reminds its patient to take their medicines on time with some additional features.
\end{abstract}

Keywords: Healthcare; Medicine box; Arduino; Wi-Fi; Alert System (or medicine reminder); Android App; SOS button

\section{INTRODUCTION}

Wouldn't it be good if we could carry a medicine box with us that would remind us to take our medicine? Not only is MediSmart, helpful to the elderly people, who are prey to old age and various diseases, but also to the youngsters who are being reckless with the matter of their health by not taking their medicines on time and thus not recovering completely. Surveys and many research studies have indicated towards adults who are elderly people and are suffering from chronic diseases but also the youngsters who have fallen prey to the busy and hectic lifestyle. This busy lifestyle has made them forget to take their medicines on time, which is essential for their healthy recovery from their diseases. MediSmart proposes a solution to this distress. It is a smart, IOT based medicine or pillbox that reminds its patient, with alert systems, to take their medicines on time some other additional features. MediSmart is a special Med Box that also helps patients with visually handicap, like blind or ocular impaired and even partially deaf people. It uses both visual and audio aids to guide the patients. It also uses an LCD display to indicate the name of the prescribed tablets to be taken. Taking medicines is one of the important aspects for maintaining good health and thus MediSmart also provides a feature that notifies the patient via the app that the medicine box is empty and needs to be refilled so that they can take their medicines on time without any delay. MediSmart is a user-friendly medicine box that fosters not only good health but also the security of the patient. It provides an SOS button that would immediately send an alert message or call to notify their respective doctors or caretakers, in case of any emergencies. MediSmart: Better health with IOT based Med Box, as the motto says MediSmart wants the patient to not only be healthy but also safe.

\section{LITERATURE SURVEY}

\section{A. Related Work}

Multiple kind of research, have been conducted in the field of Internet of Things and its applications. One of them is the smart medicine box. As the name suggests, it is fundamentally a medicine box that is equipped with smart functionalities to make the task of the user easier and to assist the user in any way possible, in context of medication intake. Many research papers have proposed various solutions to this innovative idea.

Matrix bar code technique, as used in [1] where the matrix bar code is printed on the medicine bag provided by the hospital. This matrix bar code includes the patient name, patient identification (ID), hospital identification (ID), medicine name, medicine ID, medication time, and other related information about the medicine bag [1]. It needs to be scanned through a camera secured inside the medicine box which confirms the intake of the medicine by the patient. The patient is reminded by an alarm system where the buzzer sets off at the scheduled time and the patient then needs to take the medicine bag out of the box and scan the matrix bar code to confirm that they have taken the medicine. If the patient does not take the medicine, the pillbox will remind it later again and using the user interface it guides the patient to take the right medicine [1]. This is a very effective way of ensuring timely medication intake.

Smart medicine box can also be assigned to multiple people uses different RFID tags [2] for different users. These RFID tags, with the help of RFID reader, are used to recognize the respective users so that the medicines can be given 
out. Just like the other smart medicine boxes, it provides with an alarm system [2] that reminds the patient to take their medicines. A Web page [2] is used to remotely monitor the medicine schedule followed by a patient and upload the changes if any are found after comparing the variations in medicine consuming activity in scheduling, quantity, and timing of drug. The benefit of using RFID is the advantage of Encryption and Decryption that would avoid threats of data theft or misuse over the internet [2]. Also, this smart medicine box provides with the facility of 'Appointment from home', if the patient wants to visit the doctor and the doctor can schedule an appointment using the RFID to identify the patient [2].

Smart Pillbox (SPB) can not only useful to the patients but even for the relatives of the patient. [3] SPB features a device that is embedded with sensors in each compartment that not only transmits the signal indicating that the patient has taken the medicine but also receives remind messages that are sent to the LCD screen, displaying words or patterns or voice messages[3]. This SPB is installed with the Webduino module to achieve this two-way messaging [3] with remote relatives or caretakers [3] via the internet of things. The module first reads a sensing signal in the kit and then it is transmitted to the $\mathrm{Wi}-\mathrm{Fi}$ router via $\mathrm{Wi}-\mathrm{Fi}$ connection. The $\mathrm{Wi}-\mathrm{Fi}$ router then sends this signal to the cell phones or remote web pages [3] (LCD) via the internet. Remote relatives can send their messages to the Wi-Fi router through the internet. This message is then sent to the Webduino module [3] which sends it to the SPB for the message to display or voice playback. This system is useful for the elderly living separately from their families or at nursing homes and makes it easier to monitor the medicine intake activities from remote places.

Similar to [3], doctors can keep track of the patient via access to Cloud [4], which holds all the information of the patient. The cloud server also stores information of the everyday analysis [4] of the patient and accordingly the doctor decides if the dosage needs to be continued or not or a new course of medicines should be prescribed. Only the doctor can access the cloud which is approved by the hospital. There are three end users who have login access to the patient's medication information on the application provided [4]. There three trays for each time of the day; morning, afternoon and evening when the medicines are to be taken and the patient is reminded accordingly. This way all the three end users have a track of the patient's health progress [4].

\section{B. Proposed System}

The existing systems using matrix bar code and RFID tags are disadvantageous as every time the patient needs to scan these tags when taking their medicines which can be tedious and the cameras used can also be expensive. Another system, Smart Pillbox (SPB) uses Webduino Module which is proven to be more expensive. MediSmart takes into account these shortcomings and tries to overcome them.

MediSmart is an IOT based smart medicine box which reminds the patient to take their prescribed medicine. It provides an android application with an easy to use, user interface where user can enter their details, which forms a bridge between the end user and MediSmart. The end user needs to fill and update their personal details and the details of medicine prescriptions in each field via an Android app. The user needs to sign up to the application if they are not registered. If they are already signed up, the end user can directly login and perform further activities. These activities include filling in the details of the patient, like their name, date of birth, contact information, medical information (Ex.: name of their disease), prescription details (Ex.: name of their medicine, no. of pills, time or schedule), their caretaker's or doctor's details (Ex.: name of the doctor, contact information of the doctor).

This Android Application then updates the database and saves the inputs given by the user. It is now connected to the Arduino through Wi-Fi Connection by ESP8266 WiFi-module via TCP protocol. The following system block diagram shows the overall working of MediSmart. On the scheduled time, the Arduino receives requests via TCP protocol and then sends signals to the other components of the Medicine Box rendering the alarm system to set off. The Alarm System provides both audio and visual aid to guide the end user. The basic alarm system is set off at the scheduled time, that is, a piezoelectric buzzer. This is helpful in not only reminding the non-handicapped end users but also blind or visually impaired end users. Also, in case the end user forgets to hear this, the buzzer sounds again after a specified interval of time with higher frequency. There are LED lights that are placed on the box so that partially deaf end users can be visually alerted. There are LED lights that are placed on the box so that partially deaf end users can be visually alerted. Of course, the box needs to be in the user's vicinity. Another visual functionality provided by MediSmart is the Liquid Crystal Display that indicates the name of pills to be taken by the end user. This will guide the end users suffering from memory loss to take the right medicines as prescribed.

To ensure better health, MediSmart with the help of the Android application (version 2.1.1) alerts the end user if medicines have not been consumed. It makes use of Infra-Red sensor which when obstructed by the hand of the end user, would send a signal to the Arduino UNO (version 1.0, Atmega328P) and thus detecting whether the box has been opened or not and therefore determining if the medicine has been consumed or not. In case, the box hasn't been opened 
despite the alarm systems going off, MediSmart notifies the user via an Android application that medicine has not been taken.

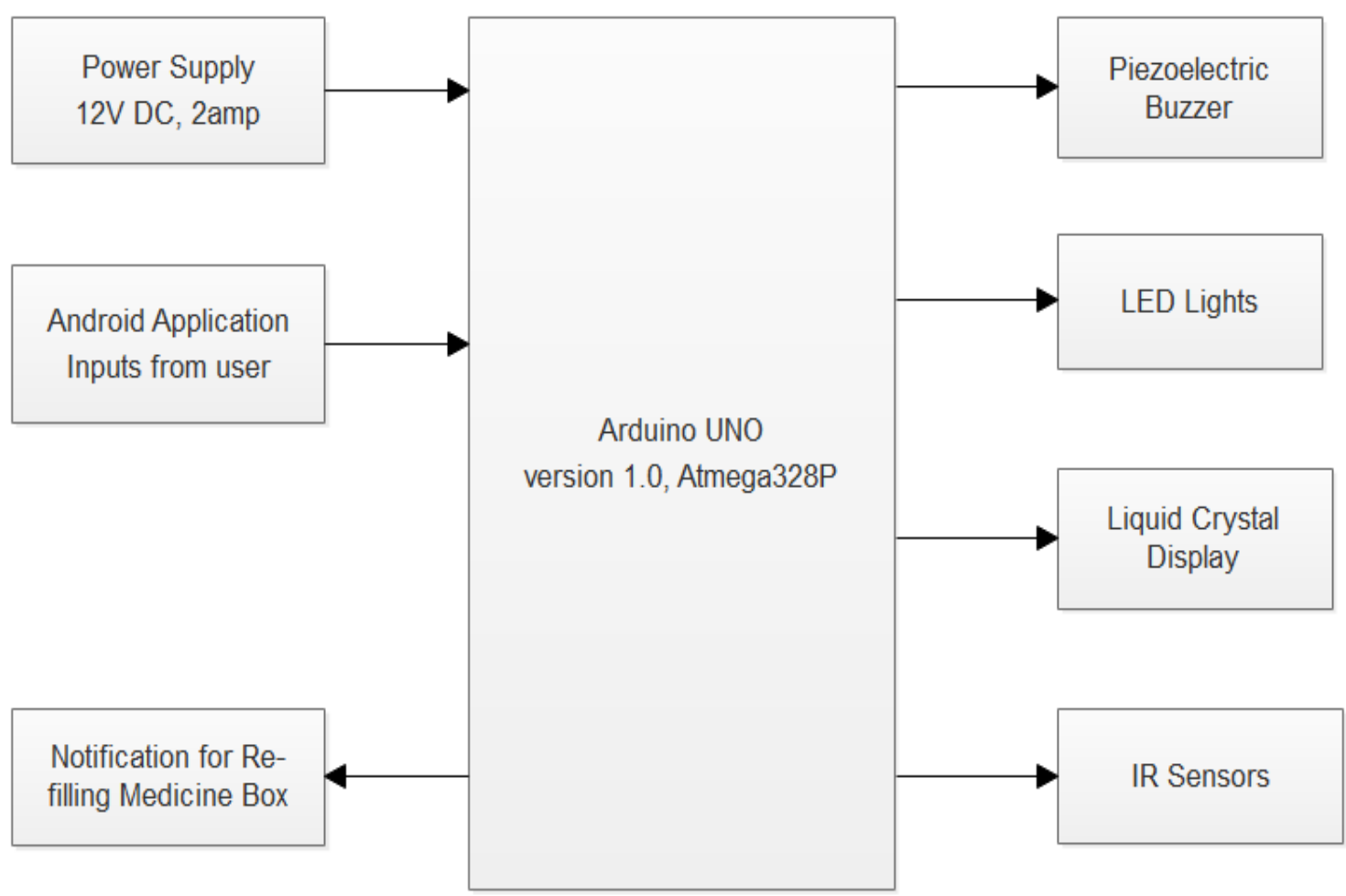

Fig. 1. The System Block Diagram of MediSmart

Taking medicines properly also includes another task at hand. For some diseases, medication could be prescribed over a span of time. Thus, medicines could be delivered as courses over short periods of time. MediSmart also facilitates in reminding the end user that it's time to refill boxes or apply for their next course, avoiding irregular intake of medicines. It uses Infra-Red sensors to detect whether the box is empty or not. MediSmart is a user-friendly system that fosters not only good health but also the security of the end user. It provides an SOS button that would immediately send an alert message or call to notify their respective doctors or caretakers, in case of any emergencies. MediSmart: Better health with IOT based Med Box, as the motto says MediSmart wants the end user not only to be healthy but also safe. This is a very important attribute of MediSmart that is truly a lifesaving boon.

MediSmart is a user-friendly IOT based device provides better health with the following advantages:

1. The buzzer sounds an Alarm to remind and aid blind and visually impaired patients.

2. LED lights glow to alert partially deaf patients.

3. Liquid Crystal Display indicates the name of the prescribed medicines to be taken at the respective time scheduled, to guide the patients to take the right amount of medicines.

4. Notification to indicate whether the medicine is taken or not.

5. Refill notification.

6. SOS button to alert respective doctors or caretakers in case of an emergency.

\section{CONCLUSION}

This paper summarizes the implementation of MediSmart with IOT based technologies. It provides a solution to the idea of smart medicine box which reminds the end user to take the medicine on time using various audio and visual alert systems. It also specifies the name of tablets prescribed that would guide the end user to follow the prescription. All these functionalities work via an Android application (version 2.1.1.) connected to an Arduino UNO (version 1.0) through Wi-Fi connection. Also, the user details can be updated via the application. MediSmart not only takes care of the end user's health but also safety. It provides a functionality of the SOS button which informs the respective doctor or caretaker about the end user's exigency. MediSmart is an ever-evolving project and has a lot of room for improvement. 
Vol. 8, Issue 4, April 2019

\section{ACKNOWLEDGEMENT}

We would like to express our sincere gratitude towards our guide, Prof. S. A. Joshi for her invaluable guidance and supervision, that helped us in our research.This work on "MediSmart: Better Health with IOT based Med Box" would not have been possible without the extensive support of our family and friends who were directly or indirectly involved in its successful completion.

\section{REFERENCES}

[1]. A Smart Pillbox with Remind and Consumption Confirmation Function,2016 IEEE $4^{\text {th }}$ Global Conference on Consumer Electronics(GCCE)

[2]. Medicine Reminder and Monitoring System for Secure Health Using IOT, Science Direct, International Conference on Information Security \& Privacy(ICISP2015),11-12December 2015(Procedia Computer Science)

[3]. Bidirectional Smart Pillbox Monitored Through Internet And Receiving Reminding Message From Remote Relatives, 2017 IEEE International Conference on Consumer Electronics - Taiwan(ICCE-TW)

[4]. Enhancing Healthcare using m-Care Box, International Conf on IEEE I-SMAC (IOT in Social, Mobile, Analytics \& Cloud) (I-SMAC 2017)

[5]. iEZ Pillbox by Infoengine Technology(shenzhen)Co.ltd

[6]. GMS Medalert 28 DAYS Days Automatic Pill Dispenser

[7]. Carlo Ghezzi, "Fundamentals of Software Engineering", $2^{\text {nd }}$ Edition, Prentice Hall India

[8]. Arshdeep Bagha, Vijay Madisetti, "Internet of Things - A hands - on approach", Universities Press.

[9]. Eric J. Braude, "Software Design: from Programming to Architecture", J. Wiley, 2004.

[10]. Ralph Moseley \& M.T. Savaliya "Developing Web" Applications", Wiley publications. 